\title{
Community Pharmacists' Roles in Optimising Opioids Therapy for Chronic Non-malignant Pain Patients: a Qualitative Study.
}

\section{Aziza Ali Alenezi}

University of Birmingham

\section{Vibhu Paudyal}

University of Birmingham Edgbaston Campus: University of Birmingham

Asma Yahyouche ( $\sim$ A.Yahyouche@bham.ac.uk)

University of Birmingham College of Medical and Dental Sciences https://orcid.org/0000-0002-33486399

\section{Research Article}

Keywords: Chronic non-malignant pain, chronic opioid therapy, medicine optimisation, community pharmacists

Posted Date: April 6th, 2021

DOI: https://doi.org/10.21203/rs.3.rs-378470/v1

License: (c) (i) This work is licensed under a Creative Commons Attribution 4.0 International License. Read Full License 


\section{Community pharmacists' roles in optimising opioids therapy for 2 chronic non-malignant pain patients: a qualitative study.}

Aziza Alenezi, ${ }^{\text {a }}$ Vibhu Paudyal, ${ }^{a}$ Asma Yahyouche, ${ }^{\text {a* }}$

${ }^{a}$ University of Birmingham, School of Pharmacy, Institute of Clinical Sciences, Edgbaston

, Birmingham B15 2TT, UK

Abstract.

Background: Opioids are currently widely used to manage chronic non-malignant pain, but there is a growing concern about harm resulting from opioid misuse, and the need for medicine optimisation, in which pharmacists could potentially play a key role.

Objective: This study seeks to identify challenges to community pharmacists' role in optimizing prescribed opioids for Chronic Non-Malignant Pain (CNMP).

14 Setting: Community pharmacies in the UK.

Method: Semi-structured interviews based on the Theoretical Domains Framework were conducted with 20 community pharmacists recruited through professional networks and analysed thematically.

Result: Pharmacists perceive themselves as guardians of patients' welfare and aspire to contribute to prescribed opioid optimisation. However, they are challenged by the lack of relevant training, inadequate time and resources, infrastructural and systemic constraints (such as repeat prescribing and prescription delivery services, lack of access to medical records and information about diagnosis), personal factors, including communication with doctors, and relationship with patients.

Conclusion: The role of community pharmacists in optimising chronic opioid therapy is neither well-defined nor implemented in the UK. Utilisation of their potential skills and knowledge in this area requires an appropriate training curriculum, tackling the infrastructural and systemic constraints, support and resources to facilitate pharmacists' engagement in patient monitoring and education. The findings in this study can contribute to inform policy makers with potentials to enhance pharmacists' role in opioids therapy optimisation and, hence, ensure patients' safety when using prescribed opioids.

Keywords: Chronic non-malignant pain, chronic opioid therapy, medicine optimisation, community pharmacists. 


\section{Introduction}

40 Chronic pain is defined as pain that persists for more than three months [1]. Data suggests that up

41 to $19 \%$ of the population in Europe and $46 \%$ in the UK suffer from chronic non-malignant pain

42 (CNMP) [2]. Managing CNMP is costly to health services. The direct cost of prescribed

43 analgesics in the UK reached $£ 537$ million in 2016 , while the indirect cost in lost productivity

44 was estimated as $£ 10.7$ billion between 2013-2014 [3].

45 Opioids have long been used in pain management. However, concerns about chronic opioid

46 therapy (COT) effectiveness, safety, and abuse in CNMP have grown, especially with the

47 alarming death rate related to medical and illicit use of opioids in North America [4]. This has

48 led to uncertainty about the future place of opioids in treating CNMP [5]. The UK reportedly has

49 the highest rate of prescribed opioid use in Europe. Moreover, half of the drug-related deaths in

50 England involve an opiate. For instance, fentanyl-related deaths increased from eight in

512008 to 135 in 2017[6]. However, the possible direct link between prescribing practices

52 (including any potentially inappropriate prescribing) and opioid-related harm and deaths is

53 under-researched [7]. The assurance of patients' safe and effective use of medicine is a challenge

54 for healthcare professionals. Medicine optimisation, which emphasises safe, effective and

55 efficient medicine use, is becoming a key priority in the UK's National Health Service (NHS) for

56 the management of long-term conditions [8]. Medicines management which is primarily led by

57 pharmacy teams and focuses on the system's process, could facilitate medicine optimisation [9].

58 Literature suggests that a well-structured CNMP management programme led by a

59 multidisciplinary team is critical to optimise COT use and improve CNMP patients' quality of 
60 life [10]. Community pharmacists can play an essential role in the use of Prescribed Opioids

61 (PO) for these patients as it is estimated that $90 \%$ of the English population live within a 20-

62 minute walk of a community pharmacy [11]. An expansion of community pharmacists' role to

63 deliver more clinical services has been supported by the NHS in a five-year deal from 2019 until

642024 that sets out a vision for that role and guarantees community pharmacies' funding levels

65 through a structured framework [12]. Community pharmacists are well positioned to deliver

66 many services that can lead to optimisation of opioids use in CNMP, including screening patients

67 for diversion, monitoring potential problematic PO use, educating patients about opioid-related

68 risks and treatment referrals for patients who are suffering from Opioid Use Disorder (OUD).

69 Studies in the USA and the UK suggest community pharmacists have constructive attitudes

70 toward pharmacy-based screening and intervention to reduce substance use disorder [13, 14].

71 However, little is known about community pharmacists' experiences as the literature that focuses

72 on their role in relation to optimisation of prescribed opioids and addresses the pharmacists'

73 perspective in the UK is sparse. Surveys in the USA show that community pharmacists are

74 facing enormous challenges, and they are willing to expand their role in optimising opioid use

75 for CNMP.

76 This study aimed to undertake a theoretically informed exploration of community pharmacists'

77 current and future roles and associated barriers in optimising opioids therapy for chronic non-

78 malignant pain patients.

\section{$79 \quad$ Ethics approval}


80 Ethical approval was granted by the Science, Technology, Engineering and Mathematics Ethical

81 Review Committee of the University of Birmingham. Written informed consent was obtained

82 from all participants.

\section{Method}

\section{Study design}

85 This study used qualitative study design. Data was obtained via semi-structured interviews based

86 on the Theoretical Domains Framework (TDF) to explore the perspective of participating

87 community pharmacists. The TDF was developed from a synthesis of psychological theories to

88 help apply theoretical approaches to behaviour change interventions. TDF domains represent

89 environmental, cognitive and social factors that may affect behaviour (Table 1).

90 Insert the table1 here.

$91 \quad$ Sample

92 Community pharmacists working in various cities in England were recruited through

93 dissemination of invitation using professional network of the research team including community

94 pharmacy forums, a large chain of community pharmacies in England and through acquaintance

95 of the research team. Characteristics of the participants are described in Table 2.

96 Insert the table 2 here.

97

98

99 Interview topic guide and Procedure

100 The topic guide was developed based on literature, experience of the research team and the TDF 101 [15]. It was pilot tested with two community pharmacists before a final version was agreed. The 
102 interviews were introduced with general questions about participants understanding of COT

103 optimization and how they deliver this in the community pharmacy. Then, it explored their

104 perceptions about COT optimisation for CNMP [16]. Interviews ranged in duration from 25 to

10565 minutes, mostly taking around 35 minutes. All interviews were audio-recorded and

106 anonymously transcribed verbatim. Recruitment and interviewing ceased after reaching thematic

107 saturation based on initial analysis and coding (no new themes or codes could be identified from

108 the last two interviews) [17].

\section{Qualitative data analysis}

110 Excerpts from interview transcripts were deductively coded into one or more of the 14 TDF

111 domains [18] and organised into a framework table generated in Microsoft Word®. When

112 several codes could be related to form a meaningful unit, this was deemed a theme. The relative

113 semblance of each domain to pharmacists' role in COT optimisation was assessed. A domain

114 was deemed relevant if there were frequents examples in the data or pharmacists' highlighted it a

115 a significant impact on optimisation. An inductive content analysis was then undertaken to

116 identify subthemes outside the TDF domains that were considered as barriers to COT

117 optimisation. Theme and subthemes were summarised to give an overall impression of how each

118 domain or extra domain factor may influence community pharmacists' optimisation of COT for

119 CNMP and illustrated using supporting quotations [19].

120 Results

121 Insert table 3 here.

122 Key themes are presented below with illustrative quotes. 


\section{Theme 1: The pharmacists' self-perception of their identity and social professional role}

124 A dominant theme throughout the interviews, arising from the TDF domain, Social and

125 Professional Role and Identity, was pharmacists' perception of themselves as guardians of

126 patient welfare. Some expressed this in general terms, as an intrinsic part of their professional

127 role. Others elaborated on particular safety concerns, including appropriate dosage, ensuring that

128 patients derive optimum benefit from their medication and do not suffer undesirable physical or

129 mental consequences, and looking out for signs of risk. When such concerns arose, they felt a

130 responsibility to take action. Some viewed COT optimisation as a specific responsibility, but

131 others viewed their role as secondary with the clinician having the main role as one interviewee

132 explained.

133

134 Pharmacists highlighted specific activities they performed pursuant to their safeguarding role,

135 particularly medicine use review (MURs) in which they asked about patients' use of the

136 medication and any problems they encountered explained. At these interviews, they might also

137 ask patients about their alcohol consumption and over-the-counter medications, but admitted that

138 they did not routinely raise these issues. Another safeguarding activity was the provision of

139 education and advice, including warning of possible side effects. In the event of serious

140 concerns, pharmacists reported deferring supply of medicine and referring back to the

141 prescribers, for example, to query the dose, highlight interactions with other medications, or

142 report suspicions of a patient's misuse or abuse of prescribed medication or other substances.

\section{Theme 2: Capability}

144 The theme Capability is related to the pharmacists' competencies for a potential role in COT 145 optimisation, rising from the TDF domains, Knowledge, Skills and Beliefs about Capabilities. 
146 In performing their role, pharmacists relied on their professional judgement around supply of

147 prescribed opioids and drawing on knowledge and skills derived from training and experiences

148 was deemed to be key. They displayed an understanding of the meaning of COT optimisation as

149 ensuring the balance between effective control of patients' condition and avoiding harmful side

150 effects. They highlighted opioid-related problems including dependence, tolerance and addiction,

151 as well as physical and psychological side-effects such as depression. They showed awareness of

152 other treatment options, including non-opioid medication and complementary, non-

153 pharmacological therapies that might reduce the need for PO.

154 Although a few described that they had specific knowledge of pain control, the majority reported

155 having little or no formal training on how to review opioid prescriptions and identify misuse;

156 only "one lecture or something" (CPRO1) but "no sort of course" (CPMZH1). Some, moreover,

157 admitted that their knowledge might not be up-to-date. Nevertheless, they generally felt

158 confident in dispensing PO and also suggested that, over time, they acquired an "intuition kind of

159 thing" (CPMYM) that helped them to identify warning signs of misuse, such as over-ordering,

160 early re-ordering and taking multiple medications. Despite their knowledge, skills and

161 experience, however several interviewees were pessimistic about their capability to contribute

162 effectively in COT optimisation, due to their lack of authority since "we're sort of almost the end

163 point before the patient gets their medication, so apart from the medication check, there's very

164 little I can do"(CPMZH1).

\section{Theme 3: Infrastructure and systemic constraints}

166 Pharmacists also highlighted specific constraints preventing them from playing a more direct and

167 effective role in COT optimisation. The TDF domain Environmental Context and Resources

168 constituted one of the largest bodies of responses in the data set and revealed the importance of 
169 infrastructural and systemic constraints in explaining the limited involvement of community

170 pharmacists in COT optimisation. For example, pharmacists spoke of lack of information,

171 whether guidelines, detailed patient records, or organisation statements of policy, to guide them

172 in assessing prescription appropriateness or the possible need for an intervention.

173

174 With regard to patient information, for example, it was pointed out that, although pharmacists

175 could access summary care records, in practice, they would not do it without a specific reason,

176 and in any case, the information contained might be too brief to be really helpful. Where

177 guidelines existed, their effectiveness, it was suggested, was undermined by ambiguity or

178 inconsistency.

179

180 Pharmacists also pointed to a lack of funding and resources, especially lack of time as a barrier to

181 more effective COT optimisation. They expounded on the difficulty of finding time to review,

182 monitor and educate the huge number of patients they encountered and perceived involvement in

183 COT optimisation as an additional burden on an already heavy workload. As interviewees

184 pointed out that devoting the necessary time to review each opioids prescription and having a

185 detailed conversation with every patient would not be feasible without additional staff.

187 Additionally, lack of funding was discussed as a particular issue for many.

189 Several participants suggested that opioid prescription review needed to be a commissioned,

190 funded service, to persuade pharmacies to undertake such activity, and for pharmacists to devote

191 their time to it. Currently, they reported the number of MURs performed annually was being 
192 reduced, one interviewee reported a reduction from 400 to 250 MURs a year, with further

193 reductions forthcoming. Moreover, the reviews were being limited to specific categories of

194 medication, which did not include pain management. Ideally, it was suggested, MURs should not

195 only embrace a wider range of conditions and medications that is commonly the case but also

196 should be more frequent, perhaps every six months. One experienced pharmacist suggested the

197 need for a facilitative tool such as a short questionnaire to assess alcohol intake, break-through

198 pain and the like, to guide pharmacists in collecting the information needed to inform

199 optimisation interventions.

200 A large volume of comments concerned systemic factors, whether technological systems or

201 prevailing norms of practice within the pharmacy service. Interviewees particularly noted the

202 difficulty created by repeat prescribing and prescription delivery services, which reduced contact

203 between patient and pharmacist and, hence, opportunities for education and monitoring. An

204 added difficulty, especially with OTC medications, was patients' freedom with regards to choice

205 of pharmacy and number of pharmacies visited instead, potentially enabling them to engage in

206 what one pharmacist linked to a "pub crawl" for medications, making it difficult to monitor their

207 behaviour. Moreover, the development of e-prescriptions, as a widely used component of

208 prescribing systems, was seen as of limited value in monitoring and controlling opioid

209 prescriptions and opioids use. Whilst it was acknowledged that e-prescriptions ensure legality,

210 reduce the possibility of error and create a better audit trail, overall, it was suggested, they would

211 make little difference. They do not solve the problem of patients going from one pharmacy to

212 another, and they might make it too easy for patients to obtain repeat prescriptions without

213 review, especially since it was feared, that other healthcare providers might not have time to do

214 proper checks of the prescriptions and just print it. This might highlight community pharmacists' 
215 perception of their relative powerlessness to monitor and control opioid prescribing or even,

216 sometimes, knowledge of how that role is carried out.

\section{Theme 4: Personal Factors}

218 Another sources of perceived constraints, captured by the TDF domain, Social Influences, was

219 pharmacists' position within a network of stakeholders- other healthcare providers and patients-

220 whose roles, expectations and perceptions have potential impact on pharmacists' views and

221 performance of their own role. A key issue in this regard was the difficulty for some pharmacists

222 of communication with prescribers in the event of any query. While some respondents indicated

223 that doctors were usually accessible and that queries were usually answered within a reasonable

224 time, others expressed frustration at the delays they experienced when they could not get through

225 to the prescriber and were forced to hold a prescription until the required check could be made.

226 Pharmacists also reported difficulties in COT optimisation arising from patients' attitude and

227 understanding. They pointed out that patients are understandably concerned primarily with their

228 symptoms and simply want relief from pain, so tend to be resistant to the idea of reduction and

229 put pressure on pharmacists who, in turn, are wary of upsetting a patient. They also felt unable to

230 intervene in patients right to make their own lifestyle choices who have complex individual

231 needs. Moreover, those complex individual needs require a gradual, personalised approach and

232 frequent follow-ups, in which pharmacists might contribute. By helping to manage potential

233 expectations and providing education on the advantage of deprescribing and limitation of

234 medication, its correct use, potential risk and the role of medication as one component in an

235 overall plan for pain management, they aspired to an ultimate aim of enabling patient's to take

236 more responsibility for their own pain management. 
237 Among personal factors, Emotions, such as stress or empathy for patients, emerged as of limited

238 relevance, being mentioned by only two interviewees. Instead, pharmacists were more concerned

239 with the need to address the aforementioned infrastructural and systemic challenges, in order that

240 they could play an enhanced role as part of an integrated approach to the safety of patients using

241 opioids.

\section{Discussion}

243 Four themes were identified in relation to the community pharmacists' perceptions of their role

244 in optimisation of COT. The majority of themes fall under the TDF domains. Pharmacists

245 expressed a belief that they have a limited role, if any, in relation to PO for CNMP and their role

246 is limited to over-the-counter opioid-containing medications.

247 Pharmacists in the community setting still seem to act as medication dispensers, or patient

248 educators rather than medicine optimisers. However, participants perceived themselves as

249 gatekeepers capable of minimising the misuse of OTC opioid-containing products, consistent

250 with the existing literature [20, 21,22].

251 Although pharmacists have been perceived as "medicines experts", doctors have been perceived

252 as the ultimate authority over patient care, deterring pharmacists from contributing to

253 optimisation of PO [23]. Pharmacists have been described in many studies as technically

254 oriented, compared to physicians who use more sophisticated problem-solving strategies,

255 including cognitive reasoning in ambiguous situations, reasoning from basic knowledge,

256 guidelines/ algorithms or experimental-based pattern recognition [24, 25]. Moreover, both new

257 graduate and senior pharmacists in this study mentioned that prescribing opioids and chronic

258 opioid therapy in CNMP, misuse and addiction were barely addressed in the pharmacy

259 undergraduate curriculum nor a focus of continuing education. The findings aligns with previous 
260 claims that pharmacists lack a "role" as clinical decision-makers, and not trained to take on the

261 responsibility for patient outcomes [26,27]. This highlights the importance of adopting a

262 purpose-built curriculum and new teaching methods that match the NHS aim of enhancing the

263 pharmacists' role to become more patient-centred, and ensure they understand key concepts,

264 such as addiction and physical dependence and how to use recently developed screening e.g.

265 (SIB) and/or revised risk (ORT-R) questionnaires [28].

266 This study's findings suggested some may be reluctant or lacking the readiness to be involved

267 more in prescribed opioids optimisation and screening of misuse or abuse of COT, and support

268 anecdotal report of high levels of stress and discomfort when pharmacists encountered a new

269 responsibility, particularly within the context of clinically complex, ambiguous and ethically

270 sensitive situations. For example, a qualitative study in Ontario, Canada showed that under

271 information-deficient conditions, community pharmacists avoided autonomous decision-making

272 and preferred to defer responsibility to the prescriber [26]. Indeed, many participants viewed

273 prescribed opioids optimisation as not community pharmacists' responsibility and perceived they

274 lacked the required authority, reimbursement, infrastructure and time, making COT optimisation

275 challenging and stressful. Thus, changes are needed in order for pharmacists to realise their

276 potential in this area and to optimise their contributions, including improved resources and

277 structures to enhance clinical practice in the community setting [29]. The profession needs to

278 take a lead role and actively engage in being part of the solution to this problem. For example, a

279 patient-centred, clinically focused pharmaceutical care practice model would provide an

280 excellent framework for pharmacists to provide assessments, develop care plans, conduct follow-

281 up evaluations and work collaboratively with other members of the healthcare team for patients

282 using PO [30]. 
283 In spite of the significance of the regular communication between patients and community

284 pharmacist in relation to COT and PO, most interviewees mentioned the lack of regular

285 communication with patients as a barrier to greater involvement with prescribed opioids

286 optimisation and COT. This may be attributed to the growing use of online and mail-order

287 pharmacies, as well as disability or limited mobility preventing CNMP patients from visiting the

288 pharmacy [31]. Therefore, interventions that target CNMP patients should consider mobility

289 limitations. Also, some participants mentioned that they did not discuss opioid risk as they

290 expected the prescriber to discuss it with the patients during the clinic visit. Similarly, Thakur et

291 al (2020) reported that most patients stated that they had not been told about opioids' possible

292 risks and prescribers and patients agreed that pharmacists should provide such information, while

293 the pharmacists perceived opioid risk and problematic use as sensitive issues that they avoided

294 raising with patients. More training regarding communication about sensitive issues like opioids

295 both for practising health professionals and professional development was deemed necessary

296 [31].

297 Prescription drug monitoring programmes (PDMPs) are a vital tool that provides an overview of

298 the patient's history of opioid dispensing. However, in this study, the majority of participants

299 described PDMP as "not helpful" and unlikely to eliminate PO misuse or optimise PO use, as

300 these prescriptions come from trusted sources. Also, participants highlighted some of the

301 drawbacks of using e-prescription, such as the automatic repeated prescription of opioids which

302 could be problematic. This is contrary to some North American and European health care

303 providers who are optimistic about the potential for electronic prescribing of controlled

304 substances (EPCS) to improve practice [17,32]. 
Medicines Use Review (MUR) services are designed to help pharmacists to discover

306 medication-related problems, yet participants stated that even during MURs, PO were not their

307 priority, as they usually focused on other chronic diseases such as hypertension and diabetes

308 mellitus. It may be that pharmacists prefer to focus on patients with less complicated medicine

309 regimes. Pharmacists often underrate their capability and cite a need for better patient

310 information when conducting MUR [33]. It would be reasonable for them to have access to

311 diagnosis and information on co-morbidities to increase the clinical relevance of pharmacist

312 recommendations and improve communications with other healthcare providers.

313 Research suggests that stigmatised groups such as COT, CNMP, opioid use disorder, and those

314 coming off opioids may be reluctant to seek advice from their healthcare providers, yet, in our

315 study, only one participant expressed empathy toward COT patients. Empathy lends patients a

316 sense of fulfilment and connectedness that encourages them to reveal their "true state of affairs"

317 and optimises the effectiveness of pharmacist-patient consultations but relatively neglected in

318 healthcare [34]. A systematic review found a clear connection between patients with substance

319 use disorders and negative attitudes, mostly implicit, of healthcare professionals resulting in

320 shorter periods of patient engagement or avoidance of patients [35]. Also, it is reported that once

321 a problematic medication-taking behaviour has been identified, community pharmacists may

322 avoid confronting patients, due to the sensitive nature of PO use, and the potential for patients'

323 perceived sense of stigma. Motivational interviewing training for pharmacists could be very

324 beneficial in helping patients change behaviours [36].

325 This study is not without its limitations. The convenience sample involved in this study was

326 relatively small, and the subjective findings may not be generalisable. Also, topic sensitivity may

327 have resulted in some responses bias. The findings in this study are consistent with research in 
328 other settings. The use of the TDF ensured incorporation of a wide range of factors and will

329 allow the mapping of the finding to theories of behaviour changes.

\section{Implications for practice}

331 Implications for practice include the need for a clearer definition and guidelines on the use of

332 COT in CNMP and the optimisation role f pharmacists. More education and training on CNMP,

333 CNMP and opioid use disorder are needed, consistent the with NHS vision of the expanded role

334 of pharmacists in clinical aspects of patient care. There is also a need for direction and support

335 from pharmacy organisations, for example, reimbursement, to empower pharmacists as opioid

336 risk educators and gatekeepers.

\section{Conclusion}

338 The role of community pharmacists in optimising chronic opioid therapy is neither well-defined

339 nor implemented in the UK. There is a potential to use community Pharmacist's skills and

340 knowledge to facilitate their engagement with CNMP patients using COT and involvement in

341 monitoring and education. However, there is a need for appropriate training curriculum, tackling

342 of the infrastructural and systemic constraints direction, support and resources. The findings in

343 this study research can contribute to inform policy makers with potentials to enhance

344 pharmacists' role in opioids therapy optimisation and, hence, ensure patients' safety when using

345 prescribed opioids. Nevertheless, further insight into pharmacists' potential role and factors

346 affecting it could be obtained by interviewing pharmacists in other settings such as GP surgeries

347 and hospitals, and other stakeholders such as GPs and pain specialists, pharmacy educators and

348 policymakers. There is also a need for a deeper understanding of the needs and perspectives of

349 patients themselves. 
351 
1.Treede R-D. The International Association for the Study of Pain definition of pain: as valid in 2018 as in 1979, but in need of regularly updated footnotes. Pain rReports. 2018;3(2) 22-25.

2.Fayaz A, Croft P, Langford RM, Donaldson LJ, Jones GT. Prevalence of chronic pain in the UK: a systematic review and metaanalysis of population studies. BMJ Open. 2016;6(6):e010364.

3.Arthritis UK . State of Musculoskeletal Health 2018: arthritis and other musculoskeletal conditions in numbers. Report, 2019.

4.Kunnumpurath, S., Julien, N., Kodumudi, G. et al. Global Supply and Demand of Opioids for Pain Management. Curr Pain Headache Rep 22, 34 (2018). https://doi.org/10.1007/s11916-018-0689-1

5.Manjiani D, Paul DB, Kunnumpurath S, Kaye AD, Vadivelu N. Availability and utilization of opioids for pain management: global issues. Ochsner J. 2014;14(2):208-15.

6.Gov.UK. Misuse of fentanyl and fentanyl analogues. https ://www.gov.uk/gover nment/publications/misuse-of-fenta nyl-and-fentanyl-analogues?utm_source=10a98b07-79434d7a-9156-169369bb0c71\&utm_medium=email\&utm_campa ign=govuk-notif ications\&utm_content=immediate. Accessed 10 Sept 2020.

7.Alenezi A, Yahyouche A, Paudyal V. Current status of opioid epidemic in the United Kingdom and strategies for treatment optimisation in chronic pain. IJCP. 2020 Nov 30:1-5.

8.NICE. Medicines Optimisation: The Safe and Effective use of Medicines to Enable the Best Possible Outcomes. London: National Institute for Health and Care Excellence 2015. Available from https://www.nice.org.uk/guidance/ng5 (accessed October 2012).

9.NHS England. [accessed Feb 3 2021]; The NHS Long Term Plan. 2019 Available at: https://www.england.nhs.uk/long-term-plan/

10.Alenezi, A., Yahyouche, A. \& Paudyal, V. Interventions to optimize prescribed medicines and reduce their misuse in chronic non-malignant pain: a systematic review. Eur J Clin Pharmacol (2020). https://doi.org/10.1007/s00228-020-03026-4

11.Paudyal V, Smith KG, MacLure K, Forbes-McKay K, Radley A, Stewart D. Perceived roles and barriers in caring for the people who are homeless: a survey of UK community pharmacists. Int J Clin Pharm. 2019;41(1):215-27.

12.Department of Health and Social Care. Community Pharmacy Contractual Framework for 201924. London: The Stationery Office; 2019.

13.Bach P, Hartung D. Leveraging the role of community pharmacists in the prevention, surveillance, and treatment of opioid use disorders. Addict Sci Clin Pract 2019;14(1):30.

14.Khan NS, Norman IJ, Dhital R, McCrone P, Milligan P, Whittlesea CM. Alcohol brief intervention in community pharmacies: a feasibility study of outcomes and customer experiences. Int J Clin Pharm. 2013;35(6):1178-87.

15.Atkins L, Francis J, Islam R, O'Connor D, Patey A, Ivers N, et al. A guide to using the Theoretical Domains Framework of behaviour change to investigate implementation problems. Implement Sci. 2017;12(1):77.

16.Michie S, Johnston M, Abraham C, Lawton R, Parker D, Walker A. Making psychological theory useful for implementing evidence-based practice: a consensus approach. BMJ Quality \& Safety. 2005 Feb 1;14(1):26-33.

17.Francis J JM, Robertson C, Glidewell L, Entwistle V, Eccles M. What is an adequate sample size? Operationalising data saturation for theory-based interview studies. . Psychol Health. 2010;25(1229-1245. 10.1080/08870440903194015.).

18.Kahlke, RM . Generic qualitative approaches: pitfalls and benefits of methodological mixology. Int J Qual Methods 2014;13:37-52. 
19.Hsieh HF, Shannon SE. Three approaches to qualitative content analysis. Qual Health Res. 2005;15(9):1277-88. over-the-counter co-codamol in Cornwall and Devon, UK: a cross-sectional survey. Heroin Addiction and Related Clinical Problems. 2018 Oct;20(N5):5-9.

21.Coombes, H., Cooper, R.J. Staff perceptions of prescription and over-the-counter drug dependence services in England: a qualitative study. Addict Sci Clin Pract 14, 41 (2019). https://doi.org/10.1186/s13722-019-0170-4

22.Carney, T., Wells, J., Bergin, M. et al. A Comparative Exploration of Community Pharmacists' Views on the Nature and Management of Over-the-Counter (OTC) and Prescription Codeine Misuse in Three Regulatory Regimes: Ireland, South Africa and the United Kingdom. Int J Ment Health Addiction 14, 351-369 (2016). https://doi.org/10.1007/s11469-016-9640-z.

23.Ilardo ML, Speciale A. The community pharmacist: perceived barriers and patient-centered care communication. Int J Environ Res Public Health. 2020;17(2). doi:10.3390/ijerph17020536.

24.Jacobs S, Hassell K, Seston E, et al. Identifying and managing performance concerns in community pharmacists in the UK. J Health Serv Res Policy 2013;18:144-50.

25.Power A, Johnson BJ, Diack HL, et al. Scottish pharmacists' view and attitudes towards continuing professional development. Pharm World Sci 2008;30:136-43.

26.Gregory, PA, Whyte, B, Austin, Z. How do community pharmacists make decisions? Results of an exploratory qualitative study in Ontario. Can Pharm J (Ott) 2016;149(2):90-8.

27.Power A, Johnson BJ, Diack HL, et al. Scottish pharmacists' view and attitudes towards continuing professional development. Pharm World Sci 2008;30:136-43.

28.Rickles NM, Huang AL, Gunther MB, Chan WJ. An opioid dispensing and misuse prevention algorithm for community pharmacy practice. Res Social Adm Pharm. 2019 Aug;15(8):959-965. doi: 10.1016/j.sapharm.2018.02.004. Epub 2018 Feb 21. PMID: 29525483.

29. Anderson C, Sharma R. Primary health care policy and vision for community pharmacy and pharmacists in England. Pharm Pract (Granada) [Internet]. 2020Mar.13 [cited 2020Dec.30];18(1):1870. Available from: https://pharmacypractice.org/journal/index.php/pp/article/view/1870.

30.Allemann, S.S., van Mil, J.W.F., Botermann, L. et al. Pharmaceutical Care: the PCNE definition 2013. Int J Clin Pharm 36, 544-555 (2014). https://doi.org/10.1007/s11096-014-9933-x.

31.Thakur T, Chewning B. Using role theory to explore pharmacist role conflict in opioid risks communication. Research in Social and Administrative Pharmacy. 2020 Aug 1;16(8):1121-6.

32.Ahmed Z, Garfield S, Jani Y, Jheeta S, Franklin BD. Impact of electronic prescribing on patient safety in hospitals: implications for the UK. The Pharmaceutical Journal: A Royal Pharmaceutical Society Publication. 2016;21:2017.

33.Stewart D, Whittlesea C, Dhital R, Newbould L, McCambridge J. Community pharmacist led medication reviews in the UK: a scoping review of the medicines use review and the new medicine service literatures. Res Soc Adm Pharm. 2020;16(2):111-122.

34.Jubraj B, Barnett NL, Grimes L, Varia S, Chater A, Auyeung V. Why we should understand the patient experience: Clinical empathy and medicines optimisation. Int J Pharm Pract. 2016;24(5):367-370https://doi.org/10.1111/ijpp.12268, Accessed date: 22 January 2019.

35.Hoppe D, Ristevski E, Khalil $\mathrm{H}$. The attitudes and practice strategies of community pharmacists towards drug misuse management: A scoping review. Journal of Clinical Pharmacy and Therapeutics. 2020 Jun;45(3):430-52.

36.van Boekel LC, Brouwers EPM, van Weeghel J, Garretsen HFL. Stigma among health professionals towards patients with substance use disorders and its consequences for healthcare delivery: Systematic review. Drug Alcohol Depend. 2013;131(2):2335https://doi.org/10.1016/j.drugalcdep.2013.02.018, Accessed date: 22 January 2019. 


\section{Supplementary Files}

This is a list of supplementary files associated with this preprint. Click to download.

- tablewithcodes28MarchAY2.pdf 